\title{
A Platform-Based Software Design Methodology for Embedded Control Systems: An Agile Toolkit
}

\author{
Lucas Cordeiro $^{1,2}$, Carlos Mar ${ }^{1}$, Eduardo Valentin ${ }^{1,4}$, Fabiano Cruz $^{1,4}$ \\ Daniel Patrick $^{1}$, Raimundo Barreto ${ }^{1}$, and Vicente Lucena ${ }^{3}$ \\ ${ }^{1}$ Departamento de Ciência da Computação - Universidade Federal do Amazonas (UFAM), Brazil \\ $\{c a a m, d p p$, rbarreto $\}$ dcc.ufam.edu.br \\ ${ }^{2}$ Centro de Ciências, Tecnologia e Inovação do Pólo Industrial de Manaus (CTPIM), Brazil \\ lucas@ctpim.org.br \\ ${ }^{3}$ Centro de P\&D em Tecnologia Eletrônica e da Informação (CETELI/UFAM), Brazil \\ vicente@ufam.edu.br \\ ${ }^{4}$ Instituto Nokia de Tecnologia (INdT), Brazil \\ \{eduardo.valentin, fabiano.cruz\}@indt.org.br
}

\begin{abstract}
A discrete control system, with stringent hardware constraints, is effectively an embedded real-time system and hence requires a rigorous methodology to develop the software involved. The development methodology proposed in this paper adapts agile principles and patterns to support the building of embedded control systems, focusing on the issues relating to a system's constraints and safety. Strong unit testing, to ensure correctness, including the satisfaction of timing constraints, is the foundation of the proposed methodology. A platform-based design approach is used to balance costs and time-to-market in relation to performance and functionality constraints. It is concluded that the proposed methodology significantly reduces design time and costs, as well as leading to better software modularity and reliability.
\end{abstract}

\section{Introduction}

Embedded computer systems are used in a wide range of systems from machine condition monitoring to airbag control systems. As the system complexity increases, its development lifecycle is also affected. Because of that, system development methodologies must be applied in order to manage the team size, the product requirement (scope), and to meet the project's constraints (time-to-market and costs). Furthermore, embedded software (ESW) plays an important role in this kind of system mainly due to its flexibility and consequently to overcome the time-to-market pressure.

Nevertheless, many development methodologies that are used to produce software that runs on the personal computers (PCs) are not appropriate for developing discrete control systems. These devices share common characteristics with typical embedded real-time systems, i.e. they have a data acquisition stage, the application of a complex control algorithm, followed by output of a result. The correctness of these systems depend not only on the results, but also on the time in which these results are produced [11].

Therefore, this kind of system contains very different characteristics such as dedicated hardware and software, and constraints that are not common to PCs based systems (e.g., energy consumption, execution time, memory footprint). In addition, severe coding errors such as implementation of finite-state machine (FSM), stack/memory overflow, and timing must be avoided when designing the ESW. Another important point is that some embedded control systems may put lives at risk (mission criticality) if some failure occurs. Therefore, definitively, these systems should be treated differently from the case where the only cost of failure is the project's investment.

Based on this context, we propose a platform-based software design methodology based on the agile principles such as adaptive planning, flexibility, iterative and incremental approach in order to make the development of embedded control software easier. To achieve that, this methodology is composed by practices from Software Engineering and Agile methods (Scrum and XP) which aim at minimizing 
the main problems present on the software development context (i.e. requirement volatility and risk management), and by others practices that are needed to achieve hardware and software development (i.e. platform-based design [23]). With this goal in mind, we also focus our attention on hardware-bound embedded software that imposes several challenges to the software design methodology (e.g., real-time and code size).

The remainder of this paper is organized as follows: Section 2 summarizes the related works. Section 3 is concerned with describing the proposed agile development methodology and its main components (processes, lifecycle, roles and responsibilities). Section 4 shows the application of the proposed methodology by focusing on the processes that were applied to the digital soft-starter and induction motor simulator prototypes. Section 5 shows the experimental results of our proposed methodology. Finally, section 6 summarizes this paper and identifies the next steps of this research.

\section{Related Works}

There are several works about agile development methodologies for embedded systems. However, there is an interesting paper that describes the experience of applying Agile approaches to the development of firmware for the Intel Itanium processor family [8]. In this paper, they identified the agile practices that the Intel team successfully applied, but they did not take into account the hardware related development. Moreover, this work did not mention how to address the non-functional requirements (e.g., code size and real-time) and did not provide any experimental results of their work.

Manhart and Schneider [13] also related a successful industrial experience when partially adopting agile methods in the production of software for embedded systems. Indeed, they made slight modifications in a well established software development process for the automotive branch adopting some agile elements in order to adequate their process to new needs as flexibility and high speed software production. As pointed out in the paper many other application areas may benefit from their experiments, nevertheless the authors did not present any measurement results that could prove their expectations.

A very interesting paper that describes the experience of applying agile test techniques to ESW is presented in [17]. In this paper, the authors focus on the test techniques that were applied to a mobile spectrometer, using a newly developed proprietary technology. In another paper, Koss and Langr propose some adaptations of test techniques used in object oriented (OO) programming languages to ESW written in $\mathrm{C}$ language [12]. There is also an interesting work that describes the application of Extreme Programming's test driven development to embedded systems featuring custom hardware and software designs [6]. In this paper, an agile method for testing embedded systems using existing software test frameworks and methodology is presented.

The conceptual framework proposed by Ronkainen e Abrahamsson, evaluate the possibility to use agile development practices in embedded software environment [16]. Therefore, they define requirements for new agile methods which include $(i)$ special emphasis on hardware/software architecture, (ii) refactoring must be integrated into the configuration management system, (iii) techniques to measure the code mature in different development phase, and (iv) techniques to design test cases that take into account not only the correctness but also the timeliness of the application. Although this paper is totally conceptual, the requirements for new agile methods served as basis for our proposed methodology.

Vicentelli and Martin propose a rigorous methodology that aims to (i) deal with integration problems among intellectual property (IP) creators, semiconductor vendors, and design house, (ii) consider metrics to measure embedded system design, (iii) work from conception to software implementation, and (iv) favor reuse by identifying requirements for real plug-and-play operation [23]. Nevertheless, they did not provide any concrete guidance and they rely on abstract rules of thumb only. Although the methodology proposed by them is totally conceptual, it also served as basis for the development of our proposed methodology.

The hardware/software co-design methodology proposed by Gajski [7] aims to develop embedded systems by formally describing the system's functionalities in an executable language rather than a natural language. The executable specification is refined through the system-design tasks of allocation, partition, and refinement. Estimators are also used in order to explore design alternatives. However, this methodology does not provide any project management activity and assumes that most of the requirements are captured before applying the partitioning algorithms.

From the point of view of embedded software design methodologies, the proposed work aims to: $(i)$ tradeoff flexibility and performance by adopting highly programmable platforms, (ii) adopt processes and practices to develop ESW that is under stringent hardware constraints, (iii) support a software driven hardware development approach through a comprehensive flow from specification to implementation, (iv) propose test techniques in a combination we have not seen before, $(v)$ make use of the iterative and incremental approach in order to offer clearly an iterative process where the designer can validate the system specification, and (vi) provide experimental results of the application of the proposed methodology in the embedded control systems domain.

The next section describes the proposed methodology 
and its main components (processes, lifecycle, and roles).

\section{Proposed Development Methodology}

The proposed methodology aims to define roles and responsibilities and provide processes, lifecycle, practices and tools to be applied in embedded real-time system projects. It contains three different processes groups that should be used during the system development: system platform, product development and management.

The system platform processes group aims to instantiate the platform for a given product. It means that the system designer must choose the system components that will be part of the architecture and API platforms from a platform library. After that, the system designer has still the possibility to customize the architecture and API platforms in order to meet the application constraints. The customization process is carried out by programming the designerconfigurable processors and runtime-reconfigurable logic integrated into the platform. The customization process is carried out by successive refinements in an iterative and incremental way into the proposed methodology.

The product development processes group offers practices to develop the application's components and integrate them into the platform. The functionalities which make up the product are partitioned into either hardware or software elements of the platform. Our partitioning algorithms used to carry out this task take into account the energy consumption, execution time, and memory size of the application's components. In addition, the partitioning technique is also applied in an iterative and incremental way. The mechanical design is also part of this processes group, but it is out of the scope of this paper.

In addition to that, this processes group aims to apply a novel test design approach in order to improve the system's coverage by stressing and covering variables and function calls in ESW. As depicted in Figure 1, this approach focuses on designing firstly the unit and functional tests before really implementing the application's code. The application's code is implemented by making use of the API platform which provides a unique abstract representation of the architecture platform through the software layer.

From the application's code, the formal translator converts it to a formal model with the purpose of allowing an embedded software engineer to verify that certain safety properties hold in the model (by using temporal logic formulas and model checking). Furthermore, if the model does not satisfy the specification then a counter-example is generated which is included into the test suite and after that it is used to adjust the application's code. Analysis of performance and energy consumption are carried out using the instruction set architecture (ISA) of the platform. This analysis is mainly based on the unit and functional tests to sim-

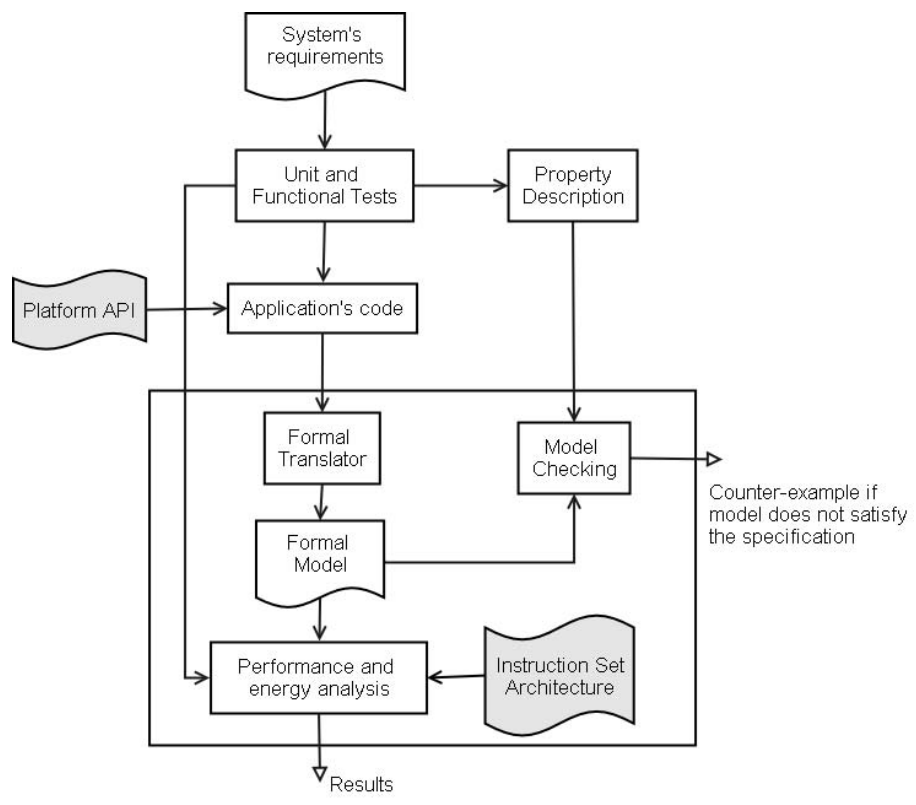

Figure 1. Test Design Approach.

ulate the system's behavior and ensure that constraints are satisfied during the development cycle.

The product scope, time, quality, and costs parameters are monitored and controlled by the product management processes group. These parameters also influence the system platform and product development processes groups. When the project starts with an infeasible project plan which needs corrective actions to be carried out then this processes group aims to get the project back on the track and ensure that the project's parameters are met. The product management processes group consists of the practices promoted by the Scrum agile method as well as the agile patterns described in $[3,18]$.

It is important to point out that the processes of the methodology were proposed in order to cover the system development lifecycle as described by [1]. Therefore, the main motivations of the proposed methodology include, but are not strictly limited to provide: (i) a full lifecycle coverage, (ii) project management activities, (iii) flexibility, (iv) means to address the non-functional requirements, $(v)$ a software driven hardware development approach, (vi) concrete guidance of the processes, and (vii) experimental results. The next subsections are concerned with describing the processes groups, roles and responsibilities, and the processes lifecycle of the proposed methodology.

\subsection{System Platform Processes Group}

The system platform processes group is composed of the following processes: product requirements, system platform, product line, and system optimization. Figure 2 
depicts the processes that are related to system platform processes group. The product requirements process aims to obtain the system's requirements (functional and nonfunctional) that are relevant to determine the system platform in which the product will be built. The platform instance process helps the development team define the system platform by making use of a set of design tools and benchmarks.

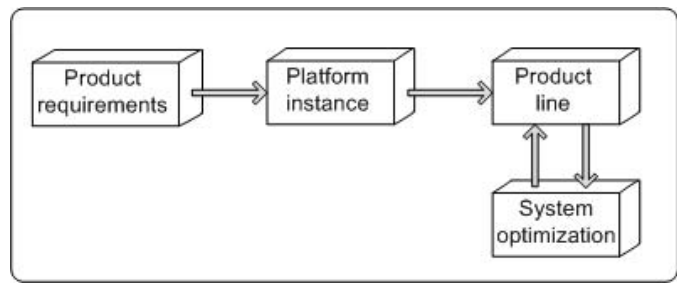

Figure 2. System Platform Processes Group.

After defining the system platform, the product line process helps the development team setup the repository in which the system platform components will be available to the product development. This process also allows the development team to implement and integrate system's functionalities into the system and release new product versions into the market. After implementing and integrating the system's functionalities into the product development line, the system optimization process provides activities to ensure that system's variables such as execution time, energy consumption, program size and data memory size satisfy the application constraints.

\subsection{Product Development Processes Group}

The product development processes group is composed of the following processes: functionality implementation, task integration, system refactoring, and system optimization. Figure 3 depicts the processes that are related to product development processes group. The functionality implementation process ensures that test cases are created for every product's functionality. This process helps increase the product quality and reduce the creation of complex functions. Moreover, it also helps create a comprehensive test suite for testing and validating that the API Platform layer will function properly for the software applications by making use of verification techniques (e.g., model checking).

The task integration process provides means to integrate new implemented functionalities into the development line of the product without forcing the other team members to work around it. The system refactoring process helps the development team identifies opportunity to improve the code and changing it without altering its external behavior. After refactoring the code, the system optimization process

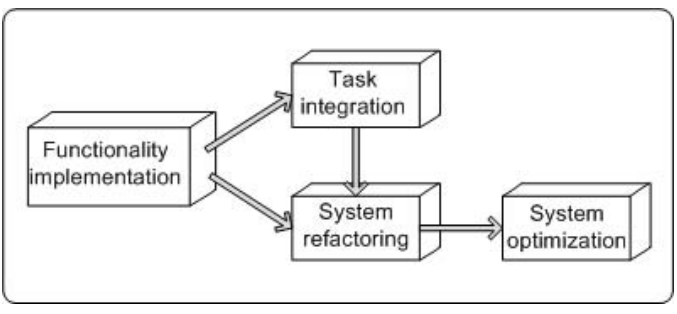

\section{Figure 3. Product Development Processes Group.}

allows the development team to optimize small part of the code by making use of profiler tools that monitor the program and tells where, for instance, it is consuming time, energy, and memory space [15]. This process guarantees that software metrics meets the system constraints.

\subsection{Product Management Processes Group}

The product management processes group is composed of the following processes: product requirements, project management, bug tracking, sprint requirements, product line, and implementation priority. Figure 4 depicts the processes that are related to product management processes group. The product requirements process (that also belongs to the system platform processes group) aims to obtain the system's requirements (functional and non-functional) that must be part of the product. The project management process allows the development team to implement the system's requirements by managing the product and sprint backlog, coordinating activities, generating system's build, and tracking the product's bug.

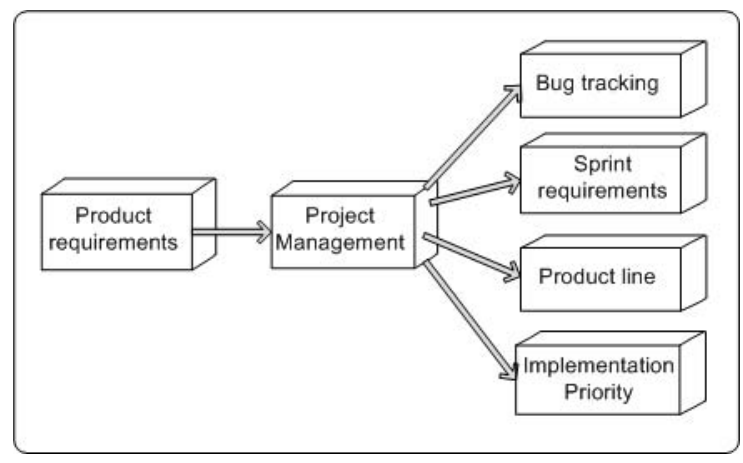

Figure 4. Product Management Processes Group.

The bug tracking process allows the product leader to manage the lifecycle of the project's issues (bug, task, and 
enhancement) and provide the needed information about the product quality through the release notes for the end user. The sprint requirements process allows the development team to analyze, evaluate, and estimate the system's functionalities before starting a new project's sprint. This information is included into the sprint backlog which will help the development team partition the system functionalities into either hardware or software before starting the sprint.

The product line process guarantees that the system functionalities implemented during the sprint will be integrated into the product development line. This process also helps the development team to release new product versions into the market. The implementation priority process helps the product leader manage any kind of interruptions that may impact the project's goals. This process guarantees that the project's tasks are 100 percent completed after initiated.

\subsection{Roles and Responsibilities}

The proposed methodology involves four different roles and the responsibility of each role is described as follows:

Platform Owner: Platform owner is the person who is officially responsible for the products that derive from a given platform. This person is responsible for defining quality, schedule and costs targets of the product. He/she must also create and prioritize the product backlog, choose the goals for the sprints, and review the product with the stakeholders.

Product Leader: Product leader is responsible for the implementation, integration and test of the product ensuring that quality, schedule, and cost targets defined by the platform owner are met. He/she is also responsible for mediating between management and development team as well as listening to progress and removes block points.

Feature Leader: Feature leader is responsible for managing, controlling and coordinating subsystem projects, pre-integration projects, external suppliers that contribute to a defined set of features. The feature leader also tracks the progress and status of the feature development (deliverables, integration and test status, defects, and change requests) and reports the status to the product leader.

Development Team: The development team which may consist of programmers, architects, and testers are responsible for working on the product development. They have the authority to make any decisions, do whatever is necessary to do (according to the project's guidelines), and ask for any block points to be removed.

If the product to be developed is small, i.e. it is composed of few components (less than 50 KLOC) and does not require other development teams to implement the product's functionalities then one product leader and the development team are enough for the product development.
On the other hand, if the product is composed by several components (more than $50 \mathrm{KLOC}$ ) and requires other development teams to implement the product's functionalities then the Feature Leader role must be involved in the processes. In this context, one product leader requires feature leaders to manage, control and coordinate components' projects. Therefore, for medium and larger projects, one product leader and several feature leaders and development teams may be involved in the processes.

\subsection{Processes Lifecycle}

The proposed agile methodology consists of five phases: Exploration, Planning, Development, Release, and Maintenance. In the Exploration phase, the customers provide requirements for the first product release. These requirements are included into the product backlog by the platform owner. After that, the platform owner and product leader estimate the requirements with no item larger than 3 person-days of effort. In this phase, the development team identifies the platform and application constraints and estimates the system's metrics based on the product backlog items. With this information at hand, the development team is able to define the system platform that will be used to develop the product in the next phases.

In the Planning phase, the platform owner and customers identify more requirements and prioritize the product back$\log$. After that, the development team spends one day to estimate the sprint backlog items and decompose them into tasks. The tasks that make up the sprint backlog must take from 1 to 16 hours to be completed. Explanatory design and prototypes may also be developed at this phase in order to help clarify the system's requirements.

In the development phase, the team members implement new functionalities and enhance the system based on the items of the sprint backlog. The daily meetings are held at the same time and place with the purpose of monitoring and adapting the activities to produce the desired outcomes. At the end of the each iteration, unit and functional tests are executed in a continuous integration build. System optimization also takes place during this phase. The last sprint provides the product to be deployed in the operational environment.

In the Release phase, the product is installed and put into practical use. During this phase, it usually involves the identification of errors and enhancement in the system services. Therefore, the platform owner and customers decide if these changes will be included in the current or subsequent release. This phase aims to deliver the release product and needed documentation to the customer. The Maintenance phase may also require more sprints in order to implement new features, enhancement and bug fixes raised in the release phase. 
The next subsections describe only a subset of processes of the proposed methodology that focuses on achieving the aims of the embedded control systems. A detailed description of all processes of our proposed methodology is public available for downloading at [4].

\section{Applying the Proposed Methodology}

This section is concerned with describing the application of the proposed methodology in the development of the digital soft-starter and induction motor simulator equipments. We chose these equipments as case studies because they impose several challenges to develop the ESW that is under stringent hardware requirements (e.g., real-time and code size) and also require a close interaction among the engineers in order to develop the products.

Both projects were split into 2 different sprints and developed by four embedded system engineers (each project had two engineers), one product leader, and one platform owner. The ESW of the digital soft-starter and induction motor simulator were implemented using the proposed methodology described in Section 3. Furthermore, these equipments were built using two development platforms.

The architecture platform for both projects contains a RS-232 serial converter, a microcontroller AT89S8253 which has an 8051-like architecture with code and data memory integrated on a chip, a real-time clock PCF8583, four channels of $\mathrm{A} / \mathrm{D}$ converter and one channel of $\mathrm{D} / \mathrm{A}$ converter. Additionally the platform has $12 \mathrm{~KB}$ of flash memory and $32 \mathrm{~KB}$ of RAM. The communication between the converters and the ESW is carried out by the communication protocol I ${ }^{2} \mathrm{C}[19]$.

We chose this platform for both projects because it has already a set of interconnected HW/SW components that together implements a set of functionalities and decreases significantly the development speed and costs. It is important to point out that after applying the proposed methodology, the final hardware configuration of the digital soft-starter equipment dispensed one channel of D/A converter, three channels of $\mathrm{A} / \mathrm{D}$ converter, and $32 \mathrm{~KB}$ of external RAM in order to reduce production costs.

Generally speaking, the digital soft-starter is an equipment that makes use of an efficient method for starting motors. Therefore, the main system's requirements that were implemented to the digital soft-starter include: $(i)$ the system should be able to automatically control the start of the induction motor, (ii) the system should read the voltage signal provided by the sensor through an analog-to-digital converter, (iii) the PWM signal generated by the microcontroller should meet all timing requirements of the application, and (iv) the user interface of the equipment should have a keyboard and a graphical display.

In order to validate the digital soft-starter equipment and also the proposed methodology, we constructed an induction motor simulator. In general, the main characteristics implemented for our induction motor simulator include: (i) the system should simulate the behavior of the motor through a mathematical model; (ii) the system should reproduce the supplied PWM signal through I/O micro-controller ports; (iii) the system should calculate and show in the display the voltage and current values based on the PWM signals supplied by the soft-starter; and (iv) a man-machine interface (display and keyboard) should be available in the final solution.

The next subsections describe the processes of the proposed methodology and the build infrastructure.

\subsection{Process for Managing the Product Re- quirements}

This process helped us identify the market needs for the digital soft-starter product line and manage the product requirements. At the beginning of the project, we arranged a brainstorming meeting in order to capture high-level requirements of the product. After that, we created an initial product backlog with the purpose of capturing more requirements and creating a first product prototype.

The first project iteration allowed us to answer questions such as whether the technology needed for the system exists, how difficult it would be, check the platform performance from different vendors, and implement a couple of system's functionalities. In further iterations, we implemented more system's requirements by focusing on the items with highest business values (the business values range from 1 lowest to 5 highest). After that, the final configuration of our development platform would dispense unneeded components and combine everything on one board for economical production costs.

As a requirement management strategy, we put much emphasis on delivering the system's functionalities $(i),(i i)$, and (iii) in the beginning of the sprints for the digital softstarter project and system's functionalities (i) and (iii) for the motor simulator project. Delivering these functionalities with highest business value, helped our customer and platform owner get feedback on functionality earlier and allow them to spot any misunderstanding more quickly. At the end of each iteration, the product leader and customer verified if the product was still feasible or not. If the project would not be feasible then it could be canceled just after the end of the iteration (risk management).

\subsection{Process for Managing the Project}

This process helped us refine and prioritize the product backlog that contains the system's functionalities. In the sprint planning, the product leader and our customer chose 
the goals of the next sprint based on the highest business value and risks of the product backlog items. After that, we had a meeting to consider how to achieve the sprint's goals and to create the sprint backlog. The sprint backlog should contain only tasks in the 4-16 hour range in order to make the management activities easier (risk reduction).

During the system development, the sprint backlog was updated regularly as the activities were being accomplished. The product leader held two meetings per week with the team members in order to monitor and control the complexity of the tasks. These daily meetings provided a great feedback to the product leader and created the habit of sharing the knowledge. After starting the sprint, we implemented first the functional requirements and then focused on the non-functional requirements of the system. This approach helped us obtain better optimization results because we optimized the global system instead of only parts of the system which sometimes might not lead to the global optimization [5].

During this phase, system's builds were also generated on a weekly basis which helped our customer clarify the requirements and asses the risks earlier in the development process. At the end of the sprint, the product leader and development team showed the results of the work to the customers. This meeting aimed to present the product increment, technology and business situation. These artifacts helped the product leader and customers decide the goals of the next sprint. In addition, after each sprint review there was a retrospective meeting which had the purpose of collecting the best practices used in the sprint and identifying what could be improved for the next sprint.

\subsection{Process for Implementing New Sys- tem's Functionalities}

This process supported us for implementing the system's functionalities of the digital soft-starter and induction motor simulator prototypes in a systematic way. According to the business value of the systems functionalities defined in the Section 4, we started implementing the requirements responsible for generating and handling the PWM signals. In order to implement these systems functionalities, we strove to write first the unit test for each stage of computation. However, this kind of activity required certain level of experience from the embedded system engineers. Nonetheless, we had to successfully compile the unit test before really writing the functionality's code.

In order to test each computation stage of the systems' functionalities, we had to run the ESW on the PC and target platform. We used this approach throughout the development cycle in order to avoid debugging hardware and software simultaneously. By running the ESW on the PC platform, we could exercise all code paths and gain confidence in our code before running it on the target platform. Another way to gain confidence in the code is to use the JTAG debug capability.

After that, we could run the ESW on the target platform to verify the application's timeliness. For both projects, we created data files on the PC that had all possible parameters combinations that make sense for the system's functions inputs. In this way, we could provide these data to our unit tests to exercise the code's paths of the functions. The unit tests created to the digital soft-starter and induction motor simulator were developed by using the embUnit framework test tool [21].

For those software components that touch the hardware, we just used the \#if and \#else statements while running the ESW on the PC platform. Later on, we developed and run application tests on the target platform in order to stress the hardware dependent code. To implement all systems' functionalities, we followed the product's coding standard defined at the beginning of the project with the purpose of keeping consistence throughout the systems' code. An important point to take into account is that if there is the need for splitting a given system's functionality into different functions then the unit tests should be created for each system's function.

\subsection{Process for Refactoring the Code}

After implementing the system's functionalities, we identified in further sprints opportunities to improve an existing code. For instance, we identified during the digital soft-starter and induction motor simulator projects that the functionalities could be split into different modules. Moreover, we also identified duplicated code in both projects. Therefore, the application of this process led to elimination of duplicated code, reduction of the amount of system's functions, and improve the system performance. However, before improving the code for those tasks in common, we first created branches in the system repository for not breaking an existing working code.

After that, we verified if there was some need for updating the unit tests of the functionality. If there was no need to update the unit tests then we could start improving the code without altering its external behavior. After refactoring the code, we run the unit tests in order to verify if the changes were working correctly. If there was no compilation problem and the unit tests would not fail then we could integrate our changes into the product development line. After integrating the code, the regression tests could also be run in order to check if there was no compilation and semantic problems. If there was no problem then the refactoring was completed. 


\subsection{Process for Managing the Product Line}

This process allowed us to set up the system repository by populating it with the system platform components which consists of the API platform. Collection of components that comprises the architecture platform were also chosen based on the application constraint's. The repository contained only the system components that were needed to derive new product lines. In other words, the development team derived the product line (or the platform instance) from the platform just by choosing a set of components from the platform library or by setting parameters of the library's reconfigurable components.

After that, they created in the system repository the development line in which the product would be built. This product development line allowed the team members to develop and optimize the product components. In order to implement new system's tasks (which may include new requirements, enhancements and bug fixes), each team member should create a branch in the product development line. This branch would help the team member implement the task without forcing other team members to work around it. After implementing and optimizing all system's components that make up the product, the development team could create a release branch of the product with the purpose of not interfering with the current system development.

\subsection{Build Infrastructure and Tools}

Figure 5 shows our proposed build infrastructure that aims to support the processes of our proposed methodo$\operatorname{logy}$. This infrastructure allows the team members to integrate new tasks into the system and hence manage the product development line as described in Subsection 4.5. The $C V S$ repository has the purpose of controlling the system's code version. In our projects, this repository is hosted in the sourceforge website and can be accessed at [14].

Our proposed build infrastructure for continuous test and integration works as follows: (i) firstly we must check out the code that is in the repository to a local workspace. Therefore, it allows us to implement new system' functionalities, fix bugs and improve the system' code. Moreover, we are also able to generate new product' builds in the local repository. (ii) after implementing and testing the code by following the activities described in the Subsections 4.3 and 4.4, we can make the code available in the repository.

(iii) After that, the Cruise Control tool looks for code modifications in the repository. If the file date/time changes then the Cruise Control starts the build process in an automated way. If there is a compliation error then Cruise Control tool sends an e-mail to the person responsible for breaking the code. Otherwise, it generates the .hex file that will

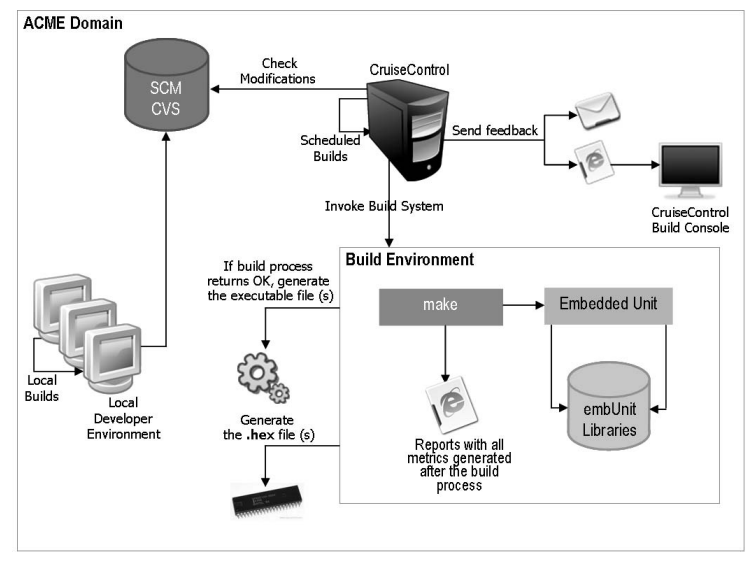

Figure 5. Continuous test and integration process.

be loaded into the flash memory of the embedded device and it also runs other tools (e.g., CCCC and EmbUnit) in order to capture the metrics and test the code. The next subsection describes the experimental results of our proposed methodology applied to the digital soft-starter and induction motor.

\section{Experimental Results}

In this section we present the results of our proposed methodology. As described in Section 4.1, we created a list of new features and requirements in order to gather all our needs (product backlog). Based on the business value, we chose from the product backlog a set of features and requirements to be implemented in the project's sprints. Therefore, we put much emphasis on delivering the systems functionalities with highest business value in the beginning of the sprints. Table 1 shows the measured effort, business value, and development speed for each sprint of both projects.

Table 1. Measured Effort (in hours)

\begin{tabular}{rrr}
\hline & Sprint 1 & Sprint 2 \\
\hline \hline Digital Soft-Starter & - & - \\
Measured Effort & 70 & 84 \\
Business value & 11 & 16 \\
Sprint Velocity & 0.1571 & 0.1904 \\
Motor Simulator & - & - \\
Measured Effort & 125 & 219 \\
Business value & 13 & 21 \\
Sprint Velocity & 0.104 & 0.0958 \\
\hline
\end{tabular}

As can be seen in Table 1, the sprint velocity of the soft- 
starter increased as the system was being developed. This situation took place due to the fact that we were still learning the involved technology, development environment, and the application domain. On the other hand, the sprint velocity of the motor simulator decreased due to the fact that a team member was moved from the soft-starter to another project. Therefore, the tasks that were allocated to him had to be transferred to an engineer of the induction motor simulator.

As mentioned in Section 4.3, we had hardware independent code and hardware specific code for the digital softstarter and induction motor simulator projects. For hardware independent code, we applied our proposed test techniques which checks not only the logic but also the timing. But for hardware specific code, we had to skip over it when running on the PC desktop. For this kind of ESW, our approach was to develop and run the test applications that aim to exercise all code paths of the function in the target platform manually. Therefore, we had approximately one test line for each two code lines using these proposed test techniques to ensure the timeliness and correctness of the prototypes.

Table 2 shows the relationship between the test and code lines of both projects. The final size of the digital softstarter and motor simulator ESW were approximately 1615 and 854 lines of code respectively. We also used the CCCC tool to count the effective source lines of code (ESLOC) of our embedded software. This tool counted 589 and 385 lines of code and 220 and 101 lines of test for the digital soft-starter and motor simulator respectively. This tool did not include blank lines, comment lines, and lines with a single brace " $\}$ ". Source files with long preambles are mainly the cause for the high percentage of non-code lines.

\begin{tabular}{rrr}
\multicolumn{3}{c}{ Table 2. Total LOC (Application and Test) } \\
\hline Project & Application & Test \\
\hline \hline Digital Soft-Starter & 1615 & 854 \\
Motor Simulator & 957 & 243 \\
\hline
\end{tabular}

The digital soft-starter and induction motor simulator embedded software had to run in a constrained environment. Our development platform had just 12KBytes of flash memory. Therefore, we used the Big Visible Chart (BVC) proposed by [2] with the purpose of tracking the memory usage and power consumption metrics. Both charts were regularly updated and kept visible in order to look for trends. Table 3 and 4 show the memory usage and power dissipation values for both projects. The current consumption was measured by connecting a multimeter in series with the energy source. The final power dissipation was then obtained by multiplying the current consumption by the supplied voltage. This power is dissipated in the whole system by the digital and analog components.

Table 3. Memory Usage (Bytes)

\begin{tabular}{rrr}
\hline Project & RAM & Flash \\
\hline \hline Digital Soft-Starter & 3631 & 3252 \\
Motor Simulator & 600 & 6398 \\
\hline
\end{tabular}

Table 4. Power Dissipation (mW)

\begin{tabular}{rr}
\hline Project & Power \\
\hline \hline Digital Soft-Starter & 855 \\
Motor Simulator & 774 \\
\hline
\end{tabular}

The test techniques described in the Section 4.3 were the suitable vehicle for software design and modularity of the digital soft-stater and motor simulator embedded software. Table 5 shows the average cyclomatic complexity of the systems. Cyclomatic complexity $(\mathrm{v}(\mathrm{G}))$ is a measure of the complexity of a module's decision structure that indicates the number of linearly independent paths [9]. Data on source code size, number of functions and cyclomatic complexity were obtained using $\mathrm{CCCC}$ tool which analyzes $\mathrm{C} / \mathrm{C}++$ files and generates a report on HTML format [20].

The final solutions of the digital soft-starter and motor simulator prototypes have approximately 35 and 31 functions in $\mathrm{C}$ code respectively. Therefore, all sprints were analyzed and the result was an average cyclomatic complexity of 1.43 and 1.61 at the end of second sprint for the digital soft-starter and the induction motor simulator. These low cyclomatic complexity levels make the white-box testing easier due to the fact that they decrease substantially the number of paths that should be tested to reasonably guard against errors. For more detail on this metric, refer to [9].

Table 5. Cyclomatic Complexity

\begin{tabular}{rc}
\hline Project & $\mathrm{v}(\mathrm{G})$ \\
\hline \hline Digital Soft-Starter & 1.43 \\
Motor Simulator & 1.61 \\
\hline
\end{tabular}

The next section concludes this work and provides goals for future research.

\section{Conclusion and Future Work}

This paper described an agile development methodology and its application in the development of the digital softstarter and induction motor simulator equipments. To illustrate the use of the processes and tools of the proposed 
methodology, we described how it was applied to develop the hardware-bound embedded software of both control systems. In these case studies, the development platform reduced substantially development time and costs of the product.

In addition, we also applied a set of test techniques in order to guarantee the timeliness and correctness of the embedded control software. These test techniques led to better software design and modularity. Therefore, we obtained 1.43 and 1.61 average cyclomatic complexity levels for the digital soft-starter and motor simulator equipments respectively. For further steps, we are researching models that can carry enough information about the ultimate physical implementation and achieve better results in terms of functional correctness.

It is important to point out that agile development methodologies are very difficult to compare with other traditional methodologies, mainly because they depend on people's pride in their work, wanting to be part of a team, and willingness to pitch in. In order to effectively compare agile and traditional development methodologies, a statistical analysis must be carried out to provide actual results in terms of productivity gains. Therefore, we are planning to perform more experimental studies where the methodology will be observed while applied in different development teams.

\section{Acknowledgements}

The authors would like to thank the support received from the Science and Technology Center for the Industrial Pole of Manaus and the Brazilian Council for Scientific and Technological Development (CNPq) by its partial financial support through project 553164/2005-8.

\section{References}

[1] P. Abrahamsson, J. Warsta, M. Siponen, and J. Ronkainen. New directions on agile methods: A comparative analysis. Proceedings of the 25th International Conference on Software Engineering, Portland, Oregon, USA, IEEE Computer Society, pages 244-254, 2003.

[2] K. Beck and C. Andres. Extreme Programming Explained Embrace Change. Second Edition, Addison-Wesley, 1999.

[3] J. O. Coplien and D. Schmidt. Organizational Patterns of Agile Software Development. First Edition, Prentice Hall, 2004.

[4] L. Cordeiro. Agile embedded software development methodology. http://www.dcc.ufam.edu.br/ lcc/methodology/, 2008.

[5] P. Cybernetica. Suboptimization Problem. Available at http://pespmc1.vub.ac.be/SUBOPTIM.html. Last visit on 26th December, 2007.
[6] M. Dowty. Test driven development of embedded systems using existing software test infrastructure. Available at http://embunit.sourceforge.net/. Last visit on 27th December 2007, 2004.

[7] D. Gajski, F. Vahid, and S. Narayan. A system-design methodology: Executable-specification refinement. European Conference on Design Automation, Paris, France, 1994.

[8] B. Greene. Agile methods applied to embedded software development. Proceeding of the Agile Development Conference (ADC'04)., 2004.

[9] S. E. Institute. Cyclomatic Complexity. Published at the Carnegie Mellon University, 2007.

[10] P. Koopman. Embedded system design issues (the rest of the story). Proceedings of the International Conference on Computer Design (ICCD96), pages 310-317, 1996.

[11] H. Kopetz. Real-Time Systems: Design Principles for Distributed Embedded Applications. Kluwer Academic Publishers, 2002.

[12] R. Koss and J. Langr. Test driven development in c. Available at http://embunit.sourceforge.net/. Last visit on 27th December 2007, 2002.

[13] P. Manhart and K. Schneider. Breaking the ice for agile development of embedded software: An industry experience report. Proceedings of the 26th International Conference on Software Engineering (ICSE04), page 3647, 2004.

[14] C. A. Mar, E. B. Valentin, F. T. Cruz, D. O. Patrick, and L. C. Cordeiro. Digital Soft-Starter and Induction Motor Simulator Code. Available at https://sourceforge.net. SourceForge, 2007.

[15] M. J. Oliveira, S. Neto, P. Maciel, R. Lima, A. Ribeiro, R. Barreto, E. Tavares, and F. Braga. Analyzing software performance and energy consumption of embedded systems by probabilistic modeling: An approach based on coloured petri nets. ICATPN 2006, LNCS 4024, pp. 261281, 2006 , page 261281, 2006.

[16] J. Ronkainen and P. Abrahamsson. Software development under stringent hardware constraints: Do agile methods have a chance? eXtreme Programming Conference, 2003.

[17] N. V. Schooenderwoert and N. Morsicato. Taming the embedded tiger - agile test techniques for embedded software. Proceedings of the Agile Development Conference (ADC'04), 2004.

[18] K. Schwaber and M. Beedle. Agile Software Development with Scrum. First Edition, Series in Agile Software Development, Prentice Hall, 2002.

[19] P. Semiconductors. The I2C-bus and how to use it. Available at http://www.mcc-us.com/i2chowto.htm. Last visit on 22th October, 2007.

[20] SourceForge. $C$ and $C++$ Code Counter. Available at http://sourceforge.net/projects/cccc. Last visit on 18th October, 2007.

[21] SourceForge. embUnit: Unit Test Framework for Embedded C Systems. Available at http://embunit.sourceforge.net/. Last visit on 18th October, 2007.

[22] V. D. Toro. Basic Electric Machines. Prentice Hall, Inc., 1990.

[23] A. S. Vicentelli and G. Martin. Platform-based design and software design methodology for embedded systems. IEEE Design and Test of Computers, 18(6):23-33, 2001. 